\title{
Transient elastography-based risk estimation of hepatitis $B$ virus-related occurrence of hepatocellular carcinoma: development and validation of a predictive model
}

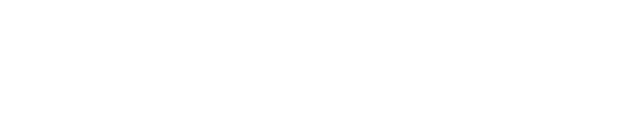

\author{
Do Young Kim ${ }^{1,2,4, *}$ \\ Ki Jun Song ${ }^{3,4, *}$ \\ Seung Up Kim ${ }^{1,2,4}$ \\ Eun Jin Yoo' \\ Jun Yong Park $1,2,4$ \\ Sang Hoon Ahn ${ }^{1,2,4}$ \\ Kwang-Hyub Han ${ }^{1,2,4}$ \\ 'Department of Internal Medicine, \\ ${ }^{2}$ Institute of Gastroenterology, \\ ${ }^{3}$ Department of Biostatistics, Yonsei \\ University College of Medicine, \\ ${ }^{4}$ Liver Cirrhosis Clinical Research \\ Center, Seoul, Korea \\ *These authors contributed equally \\ to this work
}

\begin{abstract}
Background: The purpose of this study was to develop and validate a novel transient elastography-based predictive model for occurrence of hepatocellular carcinoma (HCC).

Methods: A total of 1,250 patients with chronic hepatitis B and baseline liver stiffness values were recruited between May 2005 and December 2007. The predictive model for HCC occurrence was constructed based on a Cox proportional hazards model. We estimated baseline disease-free probabilities at 3 years. Discrimination and calibration were used to validate the model.

Results: HCC occurred in 56 patients during a median follow-up of 30.7 months. Multivariate analysis revealed that age, male gender, and liver stiffness values were independent predictors of HCC (all $P<0.05$ ), whereas hepatitis B virus DNA $\geq 20,000 \mathrm{IU} / \mathrm{L}$ showed borderline statistical significance $(P=0.0659)$. We developed a predictive model for HCC using these four variables, which showed good discrimination capability, with an area under the receiver operating characteristic curve (AUROC) of 0.806 (95\% confidence interval $0.738-0.874$ ). We used the bootstrap method to assess discrimination. The AUROC remained largely unchanged between iterations, with an average value of 0.802 (95\% confidence interval $0.791-0.812$ ). The predicted risk of occurrence of HCC calibrated well with the observed risk, with a correlation coefficient of $0.905(P<0.001)$.
\end{abstract}

Conclusion: This novel model accurately estimated the risk of HCC occurrence in patients with chronic hepatitis B.

Keywords: hepatocellular carcinoma, transient elastography, prediction, hepatitis B

\section{Introduction}

Hepatocellular carcinoma (HCC) is the fifth most common cancer globally and is a serious public health problem, particularly in association with chronic viral hepatitis. Several factors, such as chronic hepatitis B virus (HBV) or hepatitis C virus infection, cirrhosis, alcohol consumption, and obesity have been identified as risk factors for development of HCC. ${ }^{1-5}$ Moreover, the observation that over half of HCC cases and deaths are estimated to occur in far Eastern countries, including Korea, Taiwan, and China, indicates that HBV infection has a major influence on worldwide HCC epidemiology. ${ }^{6}$

Because timely and appropriate treatment and monitoring could reduce the incidence of $\mathrm{HCC}$ or detect early cancers in those with a high probability of progressing to $\mathrm{HCC}$, it is important to be able to predict $\mathrm{HCC}$ occurrence in patients with chronic hepatitis $\mathrm{B}(\mathrm{CHB})$. Both host and viral factors can affect progression of $\mathrm{CHB}$ to cirrhosis
Correspondence: Seung Up Kim Department of Internal Medicine, Yonsei University College of Medicine, 50 Yonsei-ro, Seodaemun-gu, Seoul 120-752, South Korea

Tel +82222281982

Fax +82 23936884

Email ksukorea@yuhs.ac 
or HCC in HBV-infected patients. Age, male gender, and elevated alanine aminotransferase (ALT) levels are hostrelated factors that predict occurrence of HCC. ${ }^{7}$ Virologic factors associated with the risk of $\mathrm{HCC}$ in patients with CHB include hepatitis B e-antigen ( $\mathrm{HBeAg}$ ) positivity, high serum HBV DNA levels, genotype C, and precore or core promoter mutations. ${ }^{8-12}$ However, few risk estimation and predictive models for HCC occurrence have been developed and validated in patients with CHB based on these host and virologic factors. ${ }^{10,13,14}$

The degree of liver fibrosis is also significantly related to the risk of HCC occurrence. ${ }^{15,16}$ However, to date, the severity of liver fibrosis has not been incorporated in models, because histologic assessment of liver fibrosis by liver biopsy is not always feasible in clinical practice and may cause patient discomfort and pain, and even serious complications such as bleeding or biopsy-related mortality. ${ }^{17}$ Further, sampling error and interobserver and intraobserver variability of the liver biopsy can impede its diagnostic accuracy. ${ }^{18}$ Recently, liver stiffness measurement using transient elastography (FibroScan $^{\circledR}$, Echosens, Paris, France) has been introduced as a promising noninvasive method for assessing the degree of liver fibrosis, with considerable accuracy and high reproducibility in HBV-infected patients. ${ }^{19,20}$ Stratified baseline liver stiffness values in patients with $\mathrm{CHB}$ have been demonstrated as an independent predictor of $\mathrm{HCC}$ occurrence in a recent study, where the 3-year cumulative incidence of HCC was significantly higher in patients with a higher liver stiffness value $(>8 \mathrm{kPa}){ }^{16}$

Thus, the aim of this study was to investigate whether the degree of liver fibrosis assessed by transient elastography shows a significant correlation with the risk of HCC, and if so, to construct and validate a transient elastography-based predictive model for HCC occurrence in a cohort of patients with CHB.

\section{Patients and methods Predictive model derivation}

We prospectively identified 1,250 patients with CHB who visited the outpatient clinic and received a transient elastography examination at our institution between May 2005 and December 2007. We then excluded 140 patients who did not provide written informed consent, had unreliable transient elastography data, or had insufficient clinical data for statistical analysis. Finally, the study cohort consisted of 1,110 patients, and this was used to develop the predictive model for HCC occurrence. The database for our cohort included information on patient demographics, laboratory results, and liver stiffness values at the time of enrollment. A trained medical reviewer collected patient data from medical charts. The study was approved by the independent institutional review board of Severance Hospital, Yonsei University College of Medicine, Seoul, Korea.

\section{Liver stiffness measurement}

Liver stiffness measurement was performed according to previously reported methods. ${ }^{21,22}$ Briefly, liver stiffness was measured on the right lobe of the liver through the intercostal space, with the patient lying in the dorsal decubitus position with the right arm in maximal abduction. The operator located a liver portion that was at least $6 \mathrm{~cm}$ thick and free of large vascular structures, and pressed the probe button to commence the measurement. Only one experienced technician, blind to the patient clinical data, performed the transient elastography, with results expressed in kilopascals. Only transient elastography examinations with at least 10 validated liver stiffness measurements, a success rate of at least $60 \%$, and an interquartile range to median value ratio $\leq 0.3$ were considered reliable. Any liver stiffness value that did not satisfy these conditions was considered unreliable and was excluded from further analysis. When a reliable examination was conducted, the median value of the successful measurements was selected as a representative liver stiffness value in a given patient.

\section{Diagnosis of cirrhosis and HCC}

Liver cirrhosis was clinically diagnosed as follows: platelet count $<100,000 / \mu \mathrm{L}$ and ultrasonographic findings suggestive of cirrhosis, including a blunted, nodular liver edge accompanied by splenomegaly $(>12 \mathrm{~cm})$; esophageal or gastric varices; or overt complications of liver cirrhosis. ${ }^{23,24}$ HCC was diagnosed histologically or clinically based on the guidelines of the American Association for the Study of Liver Diseases. ${ }^{25}$ Briefly, HCC was diagnosed for tumors $>2 \mathrm{~cm}$ when typical features of HCC were detected (hypervascularity and washout in the portal/venous phase) by one dynamic imaging technique and when the alfa fetoprotein level was $>200 \mathrm{ng} / \mathrm{mL}$. For nodules measuring $1-2 \mathrm{~cm}$, it was necessary to detect typical features of HCC using two dynamic imaging techniques. Ultrasonography was repeated after 3 months for tumors $<1 \mathrm{~cm}$.

\section{Statistical analyses}

All statistical analyses were conducted using SAS version 9.2 software (SAS Institute, Cary, NC, USA). The predictive model of HCC occurrence was constructed based on 
a Cox proportional hazards model, which was used to identify independent predictors of HCC occurrence with their respective $\beta$-regression coefficient, $P$-value, hazard ratio, and $95 \%$ confidence interval (CI). The median follow-up period was 30.7 (range 24.0-50.9) years, and we estimated baseline disease-free probabilities at 3 years.

$$
1-P_{0}^{\exp \Sigma \beta i X i}
$$

where $P_{0}$ is the baseline disease-free probability and $\beta i$ is the $\beta$-regression coefficient for the $i$ th independent covariate. The model was validated by discrimination and calibration. Discrimination was assessed using the receiver operating characteristic curve, area under the receiver operating characteristic curve (AUROC), sensitivity, and specificity. We calculated the predicted risk of HCC occurrence at 3 years based on our predictive model and then estimated the observed Kaplan-Meier risk of HCC to assess calibration. Kaplan-Meier estimates were plotted against the predicted risk in the group to create a calibration chart.

\section{Results}

\section{Baseline characteristics}

Table 1 shows the baseline characteristics of the cohort $(\mathrm{n}=1,110)$. The mean age was 50 years, and there were 760 males and 350 females. Cirrhosis was clinically diagnosed in $182(16.3 \%)$ patients. The median ALT level and

Table I Baseline characteristics of the cohort $(n=I, I \mid 0)$

\begin{tabular}{|c|c|}
\hline Characteristics & Values \\
\hline \multicolumn{2}{|l|}{ Demographic data } \\
\hline Age, years & $50(2 \mid-8 I)$ \\
\hline Male gender & $760(68.5)$ \\
\hline Clinically diagnosed cirrhosis & $182(16.3)$ \\
\hline Diabetes mellitus & $31(2.8)$ \\
\hline Previous or ongoing antiviral treatment & $420(37.8)$ \\
\hline Body mass index, $\mathrm{kg} / \mathrm{m}^{2}$ & $27(22-35)$ \\
\hline \multicolumn{2}{|l|}{ Laboratory data } \\
\hline Serum albumin, g/dl & $4.5(2.8-6.3)$ \\
\hline Total bilirubin, mg/dL & $0.9(0.2-3.8)$ \\
\hline Aspartate aminotransferase, IU/L & $38(12-167)$ \\
\hline Alanine aminotransferase, IU/L & $40(8-172)$ \\
\hline Prothrombin time, $\%$ & $92(45-100)$ \\
\hline Platelet count, $\times 10^{9} / \mathrm{L}$ & $160(45-520)$ \\
\hline $\mathrm{HBeAg}$ positivity & $400(36.0)$ \\
\hline High HBV DNA $\geq 20,000$ IU/L & $349(31.4)$ \\
\hline \multicolumn{2}{|l|}{ Liver stiffness measurement } \\
\hline Liver stiffness, $\mathrm{kPa}$ & $7.7(2.9-70)$ \\
\hline Interquartile range, $\mathrm{kPa}$ & I.7 (0.1-9.8) \\
\hline Success rate, $\%$ & $95(64-100)$ \\
\hline
\end{tabular}

Note: Variables are expressed as median (range) or $\mathrm{n}(\%)$. Abbreviations: $\mathrm{HBeAg}$, hepatitis $B$ e-antigen; $\mathrm{HBV}$, hepatitis $B$ virus. liver stiffness value was $40 \mathrm{IU} / \mathrm{L}$ and $7.7 \mathrm{kPa}$, respectively. $\mathrm{HBeAg}$ positivity was observed in 400 (36.0\%) patients, and HBV DNA $\geq 20,000 \mathrm{IU} / \mathrm{L}$ was observed in 349 (31.4\%) patients. Fifty-six patients developed HCC during the study period. The one-year, 2-year, and 3-year cumulative HCC incidence was $0.90 \%, 1.45 \%$, and $6.05 \%$, respectively.

\section{Multivariate Cox proportional hazards model}

First, the influence of each variable (age, gender, clinically diagnosed cirrhosis, diabetes mellitus, previous or ongoing antiviral treatment, body mass index, alpha fetoprotein, serum albumin, total bilirubin, aspartate aminotransferase, ALT, prothrombin time, platelet count, HBeAg positivity, HBV DNA, and liver stiffness value) was tested in a univariate analysis. Only age, male gender, liver stiffness value, and HBV DNA were statistically significant in univariate analysis (Table 2). A subsequent multivariate analysis was then performed using these four variables. Table 2 shows the $\beta$-regression coefficient estimates with the multivariate Cox proportional hazards model. In our cohort, age, male gender, and liver stiffness values were selected as independent predictors of HCC occurrence (all $P<0.05$ ), and HBV DNA $\geq 20,000$ IU/L showed borderline statistical significance $(P=0.0659)$. The other variables was not statistically significant $(P>0.1)$.

\section{Development of a predictive model of HCC occurrence}

In addition to the three independent variables (age, male gender, and liver stiffness value), HBV DNA $\geq 20,000 \mathrm{IU} / \mathrm{L}$ was also incorporated as a constituent variable to develop the predictive model for HCC occurrence, which has been a generally accepted significant risk factor for HCC. ${ }^{9}$

Table 2 Univariate and multivariate analysis to identify independent predictors of HCC occurrence

\begin{tabular}{|c|c|c|c|c|}
\hline \multirow[t]{2}{*}{ Factor } & \multirow{2}{*}{$\frac{\text { Univariate }}{P \text {-value }}$} & \multicolumn{3}{|c|}{ Multivariate } \\
\hline & & Beta & $P$-value & $\begin{array}{l}\text { Hazard ratio } \\
(95 \% \mathrm{Cl})\end{array}$ \\
\hline Age & $<0.0001$ & 0.0531 & 0.0003 & $\begin{array}{l}1.054 \\
(1.025-1.085)\end{array}$ \\
\hline Male gender & 0.0140 & 1.106 & 0.0044 & $\begin{array}{l}3.022 \\
(I .4 \mid I-6.473)\end{array}$ \\
\hline LSM value & $<0.0001$ & 0.0486 & $<0.0001$ & $\begin{array}{l}1.050 \\
(1.034-1.065)\end{array}$ \\
\hline $\begin{array}{l}\text { HBV DNA } \\
\geq 20,000 \mathrm{IU} / \mathrm{L}\end{array}$ & 0.0465 & 0.5097 & 0.0659 & $\begin{array}{l}1.665 \\
(0.967-2.866)\end{array}$ \\
\hline
\end{tabular}

Abbreviations: $\mathrm{HCC}$, hepatocellular carcinoma; $\mathrm{Cl}$, confidence interval; LSM, liver stiffness measurement; $H B V$, hepatitis $B$ virus. 
This newly developed predictive model showed fairly good discrimination capability, with an AUROC of 0.806 (95\% CI 0.738-0.874, Figure 1).

$$
\begin{gathered}
\text { Probability }=1-P^{\mathrm{A}} \\
\mathrm{A}=\exp (0.05306 \times \text { age }+1.106 \times \text { male gender } \\
+0.04858 \times \text { liver stiffness values }+0.50969 \\
\times \text { HBV DNA } \geq 20,000 \mathrm{IU} / \mathrm{L})
\end{gathered}
$$

\section{Discrimination and calibration}

We used the bootstrap method, in which 1,000 random samples drawn with replacement from the original data set and the coefficients were recalculated in each bootstrap sample. The AUROCs remained largely unchanged between iterations, with an average AUROC of 0.802 (95\% CI 0.791-0.812). We plotted a calibration chart for predicted and observed risk of HCC occurrence (Figure 2). The predicted risk of HCC occurrence calibrated well with the observed risk, with a correlation coefficient of $0.905(P<0.001)$.

\section{Discussion}

This prospective cohort study was undertaken to develop a clinically useful predictive model for HCC occurrence in patients with CHB using a single tertiary hospital-based cohort from South Korea, and the resulting predictive model was validated internally.

Although extensive studies have identified risk factors associated with $\mathrm{HCC}$ occurrence in patients with $\mathrm{CHB}$, few mathematically modeled risk estimations have been proposed. ${ }^{8,10,14,26,27}$ The use of hard-to-obtain variables, such

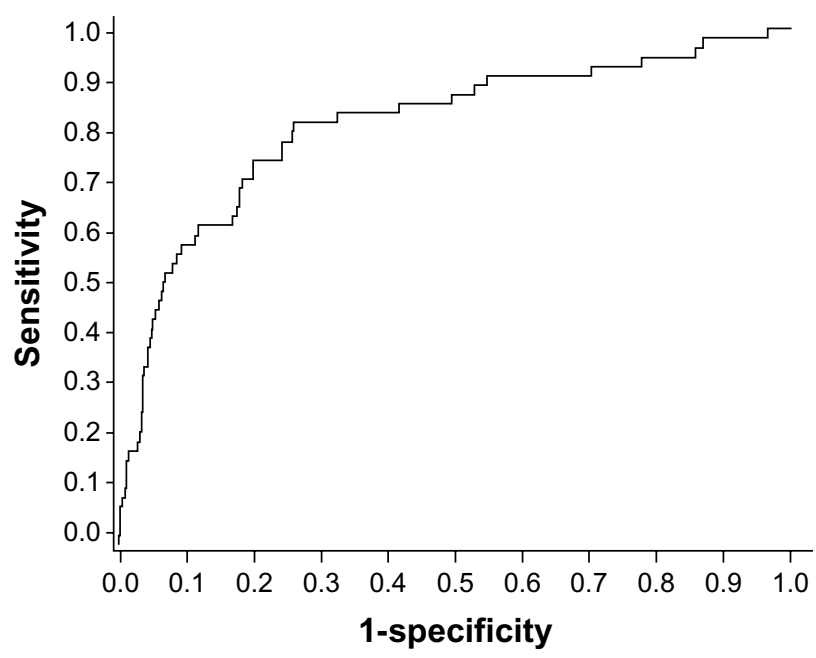

Figure I Receiver operating characteristics curve of the model. When we used the bootstrap method to assess discrimination, AUROCs remained largely unchanged between iterations, with an average AUROC of 0.802 ( $95 \%$ confidence interval $0.791-0.812$ ).

Abbreviation: AUROC, area under the receiver operating characteristic curve. as $\mathrm{HBeAg}$ and specific HBV mutations (precore or core promoter), as constituent variables or selecting naïve patients for antiviral treatment to develop a predictive model for HCC occurrence had made it difficult to apply universally to all patients infected with HBV. ${ }^{14}$ In this regard, our new model has the advantage that four simple, not exhaustive, noninvasively obtainable, and objective variables of age, male gender, HBV DNA, and liver stiffness values were included. Although statistical significance was not identified, even in the univariate analysis, we tried to adjust the influence of antiviral treatment. Finally, our new predictive model for HCC occurrence was accurate and reliable, with an AUROC of 0.806 , similar to that of a study by Yang et al in which the AUROC of the risk scores was $0.811,{ }^{10}$ and with a predicted risk correlating well with observed risk (correlation coefficient of 0.905$)$.

The suggested formula for 3-year probability of HCC occurrence, ie, $1-P^{\mathrm{A}}(\mathrm{A}=\exp [0.05306 \times$ age $+1.106 \times$ male gender $+0.04858 \times$ liver stiffness value $+0.50969 \times \mathrm{HBV}$ DNA $\geq 20,000 \mathrm{IU} / \mathrm{L}]$ ), is a revision of what we have previously reported, in which age, gender, cirrhosis, HBV/ hepatitis $\mathrm{C}$ virus infection, ALT, alfa fetoprotein, and alcohol consumption were included as variables. ${ }^{27}$ It may be argued that cirrhosis, which has been the single most important risk factor for initiating a surveillance program to detect HCC occurrence early and was selected as a significant predictor of HCC occurrence in univariate analysis of our study, should be included in this model. However, a histologic diagnosis of cirrhosis is not feasible in clinical practice, and no established and sufficiently validated clinical diagnostic criteria for cirrhosis are available, which is the reason why cirrhosis has not been incorporated into several previous risk prediction models for HCC occurrence. Further, increasing evidence has indicated that liver stiffness values, measured objectively by transient elastography, correspond accurately to the severity of liver fibrosis in both HBV and hepatitis $\mathrm{C}$ virus-related chronic liver diseases and that baseline liver stiffness values in patients with $\mathrm{CHB}$ predict $\mathrm{HCC}$ occurrence better than clinical diagnostic criteria of cirrhosis. ${ }^{23,24}$ All these results help to explain the superiority of liver stiffness values for diagnosing compensated cirrhosis. Thus, apart from the finding that liver stiffness value, not clinically diagnosed cirrhosis, was selected as one of the independent predictors of HCC occurrence in the multivariate analysis, our model might have been powered by liver stiffness values which are noninvasive and more reproducible than liver biopsy with a potential sampling error and interpretational variability, and more reliable and accurate than clinical diagnostic criteria 


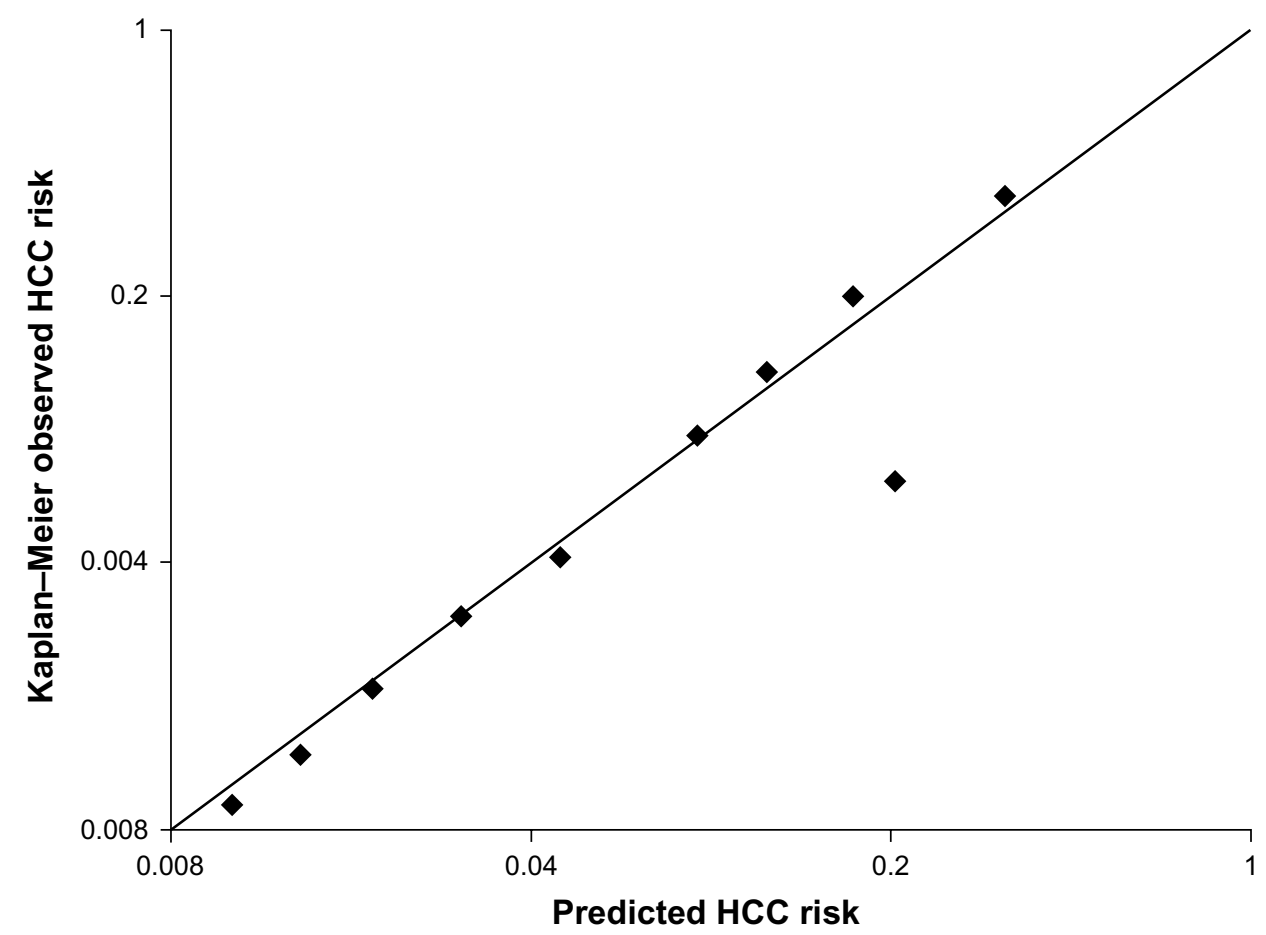

Figure 2 Calibration chart for predicted versus observed risk of occurrence of HCC. The predicted risk of occurrence of HCC calibrated well with the observed risk, with a correlation coefficient of $0.905(P<0.001)$.

Abbreviation: HCC, hepatocellular carcinoma.

for cirrhosis without sufficient validations. In addition, when the liver stiffness value was removed from our model, AUROC declined significantly from 0.806 to 0.713 (95\% CI 0.689-787; $P=0.0296$ ), which clearly indicates that the liver stiffness value can enhance the performance of our prediction model for HCC occurrence.

In addition to cirrhosis, ambiguous variables such as ALT, alfa fetoprotein, and alcohol, were removed in our revised model. Although several studies have shown that these factors might be closely associated with HCC occurrence, ${ }^{2,28,29}$ fluctuations in serum ALT levels and the influence of other conditions on alfa fetoprotein levels have made these markers unreliable for predicting HCC occurrence. Further, information on the amount of alcohol consumed by each patient is still dependent solely on patient interview, without any objective measure of alcohol consumption, and no definition of heavy alcohol consumption exists.

The finding that $\mathrm{HBeAg}$ status was not a risk factor for HCC occurrence in our study is in line with previous studies from Hong Kong and Taiwan, ${ }^{9}$ but disagrees with another report from Taiwan where a risk score incorporating gender, age, ALT level, HBeAg, and HBV DNA level was developed from a community-based untreated patient cohort with CHB. ${ }^{10}$ Therefore, it would be ideal to derive a model for predicting $\mathrm{HCC}$ occurrence in patients with $\mathrm{CHB}$ using multinational data with a large sample size, considering that a heterogeneous study population even in the same country could generate different risk factors associated with HCC occurrence. Unfortunately, because we used a less sensitive hybridization capture assay for measuring HBV DNA levels, we were not able to lower the cutoff of HBV DNA levels below 20,000 IU/mL during the study period.

Finally, antiviral treatment was not selected as an independent predictor of HCC occurrence. This result can be explained by the current antiviral treatment protocol for patients with active hepatitis to suppress viral replication and $\mathrm{HCC}$ occurrence according to most guidelines of the American Association for the Study of Liver Diseases, European Association for the Study of the Liver, and Asia Pacific Association for the Study of the Liver. ${ }^{30-32}$

Our predictive model for HCC occurrence will be useful for several purposes in clinical practice. First, it will provide an optimal surveillance strategy for patients with $\mathrm{CHB}$, which are not covered by current management recommendations. More intensive follow-up for HCC surveillance will be needed for patients assessed as having a high probability of HCC occurrence, whereas those with a low risk could be monitored with a less vigorous surveillance protocol. Second, because HBV DNA and liver stiffness values can change during the natural course of the disease, or by antiviral 
treatment, and changes in these values also have a significant influence on HCC occurrence, ${ }^{16}$ dynamic changes in the risk of HCC occurrence could be theoretically monitored by our new predictive model. If this hypothesis can be validated in future studies, patient compliance in a follow-up protocol and initiation of treatment could be enhanced by showing them the changing risk probability.

Several issues remain unresolved in our study. First, although use of transient elastography as a tool for estimation of liver fibrosis in predicting HCC occurrence is a novel approach, the use of transient elastography is still confined to referral hospitals. Thus, a limitation on the widespread use of our new model is anticipated. However, because use of transient elastography in clinical practice is currently increasing, this new predictive model in our pilot trial could be a cornerstone for future studies. Second, the risk calculation for HCC occurrence in our study was based on the probability of $\mathrm{HCC}$ occurrence at 3 years, considering the median follow-up period of our study cohort (30.7 months). Thus, more long-term follow-up seems needed to assess accurately the risk of HCC occurrence. Third, a potentially overestimated HCC incidence rate could be another issue, because our study subjects were recruited from a tertiary hospital. Last, external validation of our model in different countries with different ethnicities should be conducted for widespread practical use.

In conclusion, we have developed a simple transient elastography-based predictive model for HCC occurrence in patients with CHB. Although the performance of this new model was reasonable, based on an internal validation, the implementation of transient elastography in private clinics and external validation of our model are necessary before this new model can be used in the clinic.

\section{Acknowledgment}

This study was supported by the Liver Cirrhosis Clinical Research Center, in part by a grant from the Korea Healthcare Technology R and D Project, Ministry of Health and Welfare, Republic of Korea (A102065), the Yonsei Liver Blood Bank, and in part by a grant from Sanofi-aventis Korea. The funders had no role in the study design, data collection and analysis, decision to publish, or preparation of the manuscript.

\section{Disclosure}

The authors report no conflicts of interest in this work.

\section{References}

1. Beasley RP, Hwang LY, Lin CC, Chien CS. Hepatocellular carcinoma and hepatitis B virus. A prospective study of 22,707 men in Taiwan. Lancet. 1981;2:1129-1133.
2. Trichopoulos D, Bamia C, Lagiou P, et al. Hepatocellular carcinoma risk factors and disease burden in a European cohort: a nested case-control study. J Natl Cancer Inst. 2011;103:1686-1695.

3. El-Serag HB, Rudolph KL. Hepatocellular carcinoma: epidemiology and molecular carcinogenesis. Gastroenterology. 2007;132:2557-2576.

4. Bellentani S, Saccoccio G, Costa G, et al. Drinking habits as cofactors of risk for alcohol induced liver damage. The Dionysos Study Group. Gut. 1997;41:845-850.

5. Calle EE, Kaaks R. Overweight, obesity and cancer: epidemiological evidence and proposed mechanisms. Nat Rev Cancer. 2004;4:579-591.

6. Ferlay J, Shin HR, Bray F, Forman D, Mathers C, Parkin DM. Estimates of worldwide burden of cancer in 2008: GLOBOCAN 2008. Int $J$ Cancer. 2010;127:2893-2917.

7. Lee WM. Hepatitis B virus infection. N Engl J Med. 1997;337: 1733-1745.

8. Yang HI, Lu SN, Liaw YF, et al. Hepatitis B e antigen and the risk of hepatocellular carcinoma. N Engl J Med. 2002;347:168-174.

9. Chen CJ, Yang HI, Su J, et al. Risk of hepatocellular carcinoma across a biological gradient of serum hepatitis B virus DNA level. JAMA. 2006;295:65-73.

10. Yang HI, Yuen MF, Chan HL, et al. Risk estimation for hepatocellular carcinoma in chronic hepatitis B (REACH-B): development and validation of a predictive score. Lancet Oncol. 2011;12:568-574.

11. Chan HL, Hui AY, Wong ML, et al. Genotype C hepatitis B virus infection is associated with an increased risk of hepatocellular carcinoma. Gut. 2004;53:1494-1498.

12. Kao JH, Chen PJ, Lai MY, Chen DS. Basal core promoter mutations of hepatitis B virus increase the risk of hepatocellular carcinoma in hepatitis B carriers. Gastroenterology. 2003;124:327-334.

13. Yuen MF, Tanaka Y, Shinkai N, et al. Risk for hepatocellular carcinoma with respect to hepatitis B virus genotypes B/C, specific mutations of enhancer II/core promoter/precore regions and HBV DNA levels. Gut. 2008;57:98-102.

14. Yuen MF, Tanaka Y, Fong DY, et al. Independent risk factors and predictive score for the development of hepatocellular carcinoma in chronic hepatitis B. J Hepatol. 2009;50:80-88.

15. Park BK, Park YN, Ahn SH, et al. Long-term outcome of chronic hepatitis B based on histological grade and stage. J Gastroenterol Hepatol. 2007;22:383-388.

16. Jung KS, Kim SU, Ahn SH, et al. Risk assessment of hepatitis B virusrelated hepatocellular carcinoma development using liver stiffness measurement (FibroScan). Hepatology. 2011;53:885-894.

17. Bravo AA, Sheth SG, Chopra S. Liver biopsy. N Engl J Med. 2001;344: 495-500.

18. Rousselet MC, Michalak S, Dupre F, et al. Sources of variability in histological scoring of chronic viral hepatitis. Hepatology. 2005;41: 257-264.

19. Kim SU, Seo YS, Cheong JY, et al. Factors that affect the diagnostic accuracy of liver fibrosis measurement by Fibroscan in patients with chronic hepatitis B. Aliment Pharmacol Ther. 2010;32:498-505.

20. Wong GL, Wong VW, Choi PC, Chan AW, Chan HL. Development of a non-invasive algorithm with transient elastography (Fibroscan) and serum test formula for advanced liver fibrosis in chronic hepatitis B. Aliment Pharmacol Ther. 2010;31:1095-1103.

21. Kim SU, Kim JK, Park YN, Han KH. Discordance between liver biopsy and Fibroscan $(\mathrm{R})$ in assessing liver fibrosis in chronic hepatitis b: risk factors and influence of necroinflammation. PLoS One. 2012;7:e32233.

22. Jang HW, Kim SU, Park JY, et al. How many valid measurements are necessary to assess liver fibrosis using FibroScan(R) in patients with chronic viral hepatitis? An analysis of subjects with at least 10 valid measurements. Yonsei Med J. 2012;53:337-345.

23. Kim do Y, Kim SU, Ahn SH, et al. Usefulness of FibroScan for detection of early compensated liver cirrhosis in chronic hepatitis B. Dig Dis Sci. 2009;54:1758-1763.

24. Kim SU, Han KH, Nam CM, et al. Natural history of hepatitis B virusrelated cirrhotic patients hospitalized to control ascites. J Gastroenterol Hepatol. 2008;23:1722-1727. 
25. Bruix J, Sherman M. Management of hepatocellular carcinoma. Hepatology. 2005;42:1208-1236.

26. Chen G, Lin W, Shen F, Iloeje UH, London WT, Evans AA. Past HBV viral load as predictor of mortality and morbidity from HCC and chronic liver disease in a prospective study. Am J Gastroenterol. 2006;101: 1797-1803.

27. Han KH, Ahn SH. How to predict HCC development in patients with chronic B viral liver disease? Intervirology. 2005;48:23-28.

28. Kim HC, Nam CM, Jee SH, Han KH, Oh DK, Suh I. Normal serum aminotransferase concentration and risk of mortality from liver diseases: prospective cohort study. BMJ. 2004;328:983.
29. Hann HW, Fu X, Myers RE, et al. Predictive value of alpha-fetoprotein in the long-term risk of developing hepatocellular carcinoma in patients with hepatitis B virus infection - results from a clinic-based longitudinal cohort. Eur J Cancer. 2012;19:19.

30. EASL Clinical Practice Guidelines: management of chronic hepatitis B. J Hepatol. 2009;50:227-242.

31. Lok AS, McMahon BJ: Chronic hepatitis B: update 2009. Hepatology. 2009;50:661-662.

32. Leung N. Recent data on treatment of chronic hepatitis B with nucleos(t) ide analogues. Hepatol Int. 2008;2:163-178.

\section{Publish your work in this journal}

OncoTargets and Therapy is an international, peer-reviewed, open access journal focusing on the pathological basis of all cancers, potential targets for therapy and treatment protocols employed to improve the management of cancer patients. The journal also focuses on the impact of management programs and new therapeutic agents and protocols on

\section{Dovepress}

patient perspectives such as quality of life, adherence and satisfaction The manuscript management system is completely online and includes a very quick and fair peer-review system, which is all easy to use. Visit http://www.dovepress.com/testimonials.php to read real quotes from published authors.

\footnotetext{
Submit your manuscript here: http://www.dovepress.com/oncotargets-and-therapy-journal
} 